\section{Ehrliche Aufklärung gewünscht}

\begin{abstract}
Patienten mit fortgeschrittenem Malignom ihre infauste Prognose mitzuteilen, ist eine der Herausforderungen im onkologischen Alltag. Wie wirkt sich die Aufklärung auf das psychische Befinden der Betroffenen aus?
\end{abstract}

Eine ne ehrliche Aufklärung über Krankheit und Prognose könne Patienten helfen, ihre Situation realistisch einzuschätzen und die verbleibende Lebenszeit möglichst gut zu nutzen, so die These in einer multizentrischen Beobachtungsstudie mit 590 Patienten, die an einem soliden, metastasierten Tumor erkrankt waren. Alle waren nach mindestens einer palliativen Chemotherapie progredient und wurden bis zum Tod begleitet. $\mathrm{Zu}$ Beginn wurden sie gefragt, ob ihnen der Onkologe eine Einschätzung der Prognose gegeben hatte, und gebeten, ihre Lebenserwartung selbst einzuschätzen sowie Fragen zur ArztPatienten-Beziehung, seelischen Belastungen und ihren Wünschen bezüglich einer Sterbebegleitung zu beantworten.

299 der Patienten $(51 \%)$ gaben eine Selbsteinschätzung ihrer Lebenserwartung ab, jedoch erinnerten sich nur 17,6\% an eine klare Auskunft dazu von ihrem Arzt. Die Selbsteinschätzung dieser Patienten war realistischer als die derjenigen, mit denen kein aufklärendes Gespräch stattgefunden hatte (geschätzte Lebenserwartung median 12 vs. 48 Monate; $\mathrm{p}<0,001)$. Die aufgeklärten Patienten konnten eher anerkennen, dass sie unheilbar krank waren ( 60,8 vs. $28,7 \%$; adjustierte Odds Ratio [OR] 3,21; $95 \%$-Konfidenzintervall [95\%-KI] 2,00-5,15).

Im Median lebten die Patienten noch 5,4 Monate - über die Prognose aufgeklärte 4,8 Monate, die anderen 5,8 Monate $(\mathrm{p}=0,07)$. Von 252 der 299 Patienten, die selbst ihre Prognose eingeschätzt hatten, lagen Überlebensdaten vor: $86,5 \%$ hatten ihre Lebenserwartung zu hoch eingeschätzt, $45,6 \%$ um mehr als 2 Jahre, $29 \%$ sogar um mehr als 5 Jahre.

Es fand sich eine Assoziation zwischen der Aufklärung und trauriger oder depressiver Stimmung. Nach Kontrolle für andere Faktoren wie Alter, Geschlecht, Herkunft, Krebsart und Performancestatus wurde diese jedoch nichtsignifikant. Eine höhere Wahrscheinlichkeit für Ängste oder Depression infolge der Auf- klärung war nicht zu beobachten. Patienten, die ihre Lebenserwartung höher einschätzten, neigten dazu, sich eher lebensverlängernde Maßnahmen zu wünschen.

Fazit: Die Aufklärung über die Prognose verhilft Krebspatienten zu einer realistischeren Einschätzung der verbleibenden Lebenszeit. Arzt-Patienten-Beziehung oder emotionales Befinden werden durch das Wissen um die Prognose nicht beeinträchtigt. Kathrin von Kieseritzky

Enzinger AC et al. Outcomes of Prognostic Disclosure: Associations With Prognostic Understanding, Distress, and Relationship With Physician Among Patients With Advanced Cancer. J Clin Oncol. 2015;33(32):3809-16.

\section{Kommentar von Dr. Vehling:}

Wie können ärztliche Gespräche bei fortgeschrittener Erkrankung zu einer Versorgung beitragen, die im Einklang mit den Präferenzen des Patienten steht? Diese Frage stellt sich v.a. vor dem Hintergrund der frühen Integration palliativer Versorgung und deren Ziel, die Lebensqualität zu stärken sowie aggressive Therapiemaßnahmen am Lebensende zu vermeiden. Ein Element dieser Gespräche kann die Aufklärung über die konkret verbleibende Lebenszeit sein. Diese, so wird in der Studie gefolgert, kann zu einer realistischeren Einschätzung der eigenen Lebenserwartung führen, die wiederum mit einer adäquateren Versorgung einhergeht. Jedoch handelt es sich bei der subjektiven Lebenserwartung um ein psychologisch komplexes Phänomen, das alternative Erklärungen für den gefundenen Zusammenhang zwischen genauer Prognoseaufklärung und subjektiver Lebenserwartung zulässt. So ist es auch denkbar, dass von Beginn an weniger optimistische Patienten im Gespräch häufiger aktiv eine konkrete Einschätzung der Überlebenszeit einfordern. Darüber hinaus könnten sich Patienten, die bei Befragung eine stark ausgeprägte Überschätzung der Lebenserwartung zeigten (wie dies in der Stichprobe häufig auftrat), an eine tatsächlich erfolgte Prognoseaufklärung nicht erinnern.
Besonders aufschlussreich ist jedoch ein anderes Ergebnis: Mehr als zwei Drittel der Patienten mit fortgeschrittener Erkrankung wünschten sich ein Gespräch mit ihrem Onkologen über ihre verbleibende Lebenserwartung - und zwar unabhängig davon, ob ein solches Gespräch bereits einmal stattgefunden hatte oder nicht.

Welche Motive liegen diesem Bedarf zugrunde? Interviewstudien zeigen, dass sich die Mehrheit der Patienten ein gemeinsames Abwägen von Zukunftswünschen, -hoffnungen und -sorgen vor dem Hintergrund einer begrenzten Lebenszeit wünscht. Das Ergebnis kann sowohl als Leitfaden für informierte Behandlungsentscheidungen dienen, als auch die psycholo-

\section{"Die subjektive Einschätzung der Lebenserwartung kann im Verarbeitungsprozess fluktuieren."}

gische Verarbeitung einer verkürzten Lebenserwartung stützen. Es besteht jedoch noch umfassender Forschungsbedarf bezüglich der wechselseitigen Beziehung von Arztgespräch, prognostischer Aufklärung und psychologischen Verarbeitungsprozessen am Lebensende. In neueren Konzepten wird davon ausgegangen, dass ein adaptiver Zustand in dieser Situation von "Double Awareness" geprägt ist, einem zeitlich fluktuierenden Bewusstsein für den nahenden Tod einerseits und dem Willen zu leben sowie der sinnstiftenden Gestaltung der verbleibenden Lebenszeit andererseits. Folgerichtig sollte man in Studien wie in der Praxis die realistische subjektive Einschätzung der Lebenserwartung nicht als feste Zielgröße, sondern vielmehr als beweglichen Teil eines Verarbeitungsprozesses betrachten. Die Möglichkeit eines offenen Gesprächs über den Verlauf einer terminalen Erkrankung kann Patienten in diesem Prozess unterstützen und ihnen auch helfen, wertbasierte Behandlungsentscheidungen zu treffen.

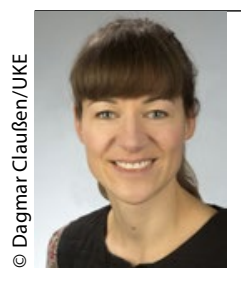

Dr. phil. Sigrun Vehling Institut und Poliklinik für Medizinische Psychologie, Zentrum für Psychosoziale Medizin, Universitätsklinikum Hamburg-Eppendorf s.vehling@uke.de 